\section{Inorganic Mass Spectrometry: Principles and Applications}

Johanna Sabine Becker

John Wiley \& Sons, Ltd.

The Atrium, Southern Gate

Chichester, West Sussex P019 8SQ, UK

ISBN 978-0-470-01200-0

Copyright (C) 2008; Hardcover, \$ 200 US, 514 pp.

Reviewed by F. C. Adams, Ph.D.

Department of Chemistry

University of Antwerp

Wilrijk, B-2610 Belgium

E-mail: freddy.adams@ua.ac.be.

Inorganic (or atomic) mass spectrometry is a very important field in elemental analysis. Its outstanding features are the possibility of determining elemental concentrations at ultratrace concentration levels. Mass spectrometric techniques for very sensitive multielemental analysis have been established for high-purity materials including metals, semiconductors, insulators, ceramics, alloys, thin films, pure chemicals, various types of environmental samples, and geological materials. One outstanding feature of mass spectrometry-the possibility of determining accurate masses and accurate abundances of stable and unstable isotopes-has grown in importance during the last years as a result of significant improvements with respect to the accuracy of isotope-ratio measurements. Applications abound for environmental monitoring, the study of isotopic variations in environmental sciences, geochemistry and geochronology, planetary science, and nuclear science.

Inorganic mass spectrometry enjoyed a widening interest over the last decade because of dramatic improvements in existing techniques, the successful commercialization of new ones, and their application to increasingly difficult analytical problems. The instrumentation of mass spectrometry improved because efficient and powerful ionization techniques, more powerful ion separation and beam handling tools, and the development of extremely sensitive ion-detection systems have emerged.

Inductively coupled plasma mass spectrometry (ICPMS) has developed into the most widely used technique today for both multielemental trace/ultratrace analysis and for isotope-ratio measurements. For the latter application-through the use of double-focusing sector field spectrometers with multicollector ion-collection devices-ICP-MS now has gradually been replacing thermal ionization mass spectrometry (TIMS) in most application areas. Laser ablation (LA) ICP-MS provides potential for the direct analysis of solids but also for surface analysis and localized or imaging analysis. Less often used techniques such as glow discharge mass spectrometry (GDMS) and secondary ion mass spectrometry (SIMS) are used for surface analysis and imaging analysis, whereas accelerator mass spectrometry (AMS) is used for the application of ultratrace isotopic abundance measurements at unmatched sensitivity.

With the growth of inorganic mass spectrometry over the years, it is important that comprehensive books become available that summarize the state of the art of the rapidly evolving techniques, the instrumentation, and the applications. There are several books on ICPMS, the most prominent among them being the deeply detailed book edited by Akbar Montaser (1998) and the less detailed accounts of Steve Hill published in 2006 (2nd edition), Simon Nelms (2005), and Howard Taylor (2002). A book with the same coverage as this one was edited by de Laeter and was published in 2001, although it misses the wide coverage of the present book. Other works such as the one edited by Barshick et al. and Adams et al. are now largely outdated. So, a comprehensive and contemporary account on this field can be considered as most welcome.

Dr. Sabine Becker has risen to this challenge and, moreover, she undertook this quite demanding task as an author, thus being able to achieve a more coherent in-depth account of the subject than what is possible with multiauthored works. The author is a respected scientist with a long experience in mass spectrometry gathered over 30 years of active and well-accepted research work. She continues to publish numerous papers on a wide range of aspects of the field and, in addition, she is the author of a number of informative review papers on particular aspects of the subject matter: isotopic analysis (J. Anal. At. Spectom., 2005), laser ablation (Int. J. Mass Spectrom., 2005), mass spectrometry of long-lived radionuclides (Spectrochim. Acta $B$, 2003), and analysis of high-purity materials (Int. J. Mass Spectrom., 2003), to mention only the most recent ones.

After a thorough discussion on the history of atomic mass spectrometric techniques, the first part of the book describes fundamentals of inorganic mass spectrometry (basic principles and developments of ion sources, ion separation systems, and ion detectors) and instrumental developments in ICP-MS, LA-ICP-MS, GDMS, SIMS, TIMS, and other mass spectrometric techniques. This part continues with practical analytical considerations for the analysis of special samples such as gaseous materials and volatile compounds and isotope ratio determinations (chapters 1-8). The second part focuses on a multitude of quite different applications including materials science, environmental science and environmental control, biology including bioengineering and medicine, food analysis, geology, geochemistry, cosmochemistry, planetary and space science, analysis of radionuclides, and forensic applications (all this in a 
well over 200 pages long chapter 9). The discussion closes with a description of cluster and ion formation in mass spectrometry (which would perhaps better have been situated with the fundamentals in the first part of the book). Appendices include tables of isotopic abundances and masses, ionization energies and atomic weights of the elements, and a list of certified reference materials from NIST and IRMM for isotopic ratio measurements. The book contains almost 2000 literature references, most of them citing work published from 2000 up to the quite recent past.

Although the concept is perhaps not directly applicable in this particular context, we can apply the SWOT technique (analysis of strengths, weaknesses, opportunities, and threats) to the desired objectives of this work as they are stated by author and publisher. For that we first need to define those objectives. The book concerns the determination of the composition of various materials on the basis of the mass spectrometric detection of stable or radioactive atoms. In that sense, a title such as "atomic mass spectrometry" would have been somewhat more appropriate than "inorganic mass spectrometry," which might lead to the false conclusion that the work concerns exclusively the analysis of inorganic materials or of organometallics. The boundaries between atomic, inorganic molecular MS, and even organic MS are now more blurred, and thus it depends on the judgment of the author to define the contents of the work. Dr. Becker decided to include speciation analysis, which depends on the hyphenated use of various separation tools (gas and liquid chromatography, size exclusion chromatography, capillary electrophoresis, etc.) and mass spectrometric detection. This is necessary because $30 \%$, perhaps even more, of the publications in this area of research are concerned with speciation analysis. With this subject definition we can now start the SWOT analysis.

$S$ for strengths. Dr. Becker's book shows tremendous strengths as a new high-quality product. It is a comprehensive and concise compilation of the body of knowledge available on the research field. As a compendium on the issue it gives insight in the state of the art of different atomic mass spectrometric techniques and should find a place in personal, academic, and corporate libraries. The book is well written, easy to read and coherent in style. The abundant illustrative material is mostly of very high quality. The publisher has also made tremendous efforts in this high-quality production: the heavy high-quality glossy paper and the attractive print makes it easy reading material. With the particular way the material on different mass spectrometric techniques is scattered over the different chapters, it is not straightforward to find back material on a particular topic, but the author did an excellent work in providing a high-quality index. As the text for a graduate course on the subject, the book seems to be a bit too detailed, but the instrumental part in chapters 1-8 might well serve as a textbook for that purpose.
$W$ : then are there any weaknesses? My major remark is that some techniques are more fully covered than others, undoubtedly reflecting the more pronounced experience of the author with certain techniques and applications than with others. For example, the coverage of SIMS and SIMS imaging is somewhat limited and the basic distinction between the static and the dynamic techniques is not put in clear evidence. The material in this book is mostly presented in a descriptive manner. The text would gain by a somewhat more critical approach toward the subject matter. Finally, the overview of what can be expected as developments and trends in the future is extremely short (barely 2 pages) and is rather uninspired.

O for missed opportunities. In the last decade, massspectrometry-based proteomics has become an indispensable analytical tool for molecular biology, cellular biology, and, lately, for the emerging systems biology. The roles that elemental mass-spectrometric detection and, particularly, ICP-MS play in this research field of "metallomics" is overall insufficiently highlighted in the book. In this field, the metabolic functions of genes (DNA and RNA) and proteins cannot be performed without the aid of various metal ions and metalloenzymes, which need to be followed sensitively. This omission must be a deliberate choice of the author, given that she and coworkers made important contributions in this particular research area. Obviously, she wanted to avoid moving too far into the now growing field of interaction between organic and inorganic analysis. Organic mass spectrometry is becoming increasingly more important in elemental speciation analysis, especially for environmental monitoring, biological metabolic research, and toxicology research; hence, a concise discussion of the main features of the organic analysis methodologies (mass spectrometric instruments and chromatographic separation tools) would have been useful.

$T$ for threats. Given the fast-moving rate of technological and scientific advancement in the field of mass spectrometry, much of the information in the book is likely to become quickly outdated. The yearly rate of publication of refereed articles on ICP-MS is now about 1000 , and this figure will increase in the future rather than decreasing or leveling off. Because this book will be of value to a wide range of users, both as a practical guide and, perhaps, as an educational device, it will be a challenge for the author to keep the material updated for further editions of this work in the coming years.

Overall, the author and the publisher did an exemplary job in the production of this outstanding and useful, extremely practical work. I would highly recommend it to anyone who is currently working in the field as a reference work but also to those interested in the potential of this methodology and who may be moving into applications of atomic MS. Moreover, at a list price of $\$ 200$ US or $€ 135$ Euro, it is a real bargain. 